\title{
Development
}

\section{Using the opinions of coronary heart disease patients in designing a health education booklet for use in general practice consultations}

\author{
Claire S. Leathem ${ }^{1}$, Margaret E. Cupples ${ }^{1}$, Mary C. Byrne ${ }^{2}$, Molly Byrne ${ }^{3}$, Mairead Corrigan ${ }^{4}$, \\ Susan M. Smith ${ }^{5}$ and Andrew W. Murphy ${ }^{2}$ \\ ${ }^{1}$ Department of General Practice and Primary Care, Queen's University, Belfast, UK \\ ${ }^{2}$ Department of General Practice, National University of Ireland Galway, Galway, Eire \\ ${ }^{3}$ School of Psychology, National University of Ireland Galway, Galway, Eire \\ ${ }^{4}$ Medical Education, Queen's University, Belfast, UK \\ ${ }^{5}$ Public Health and Primary Care, Trinity College, Dublin, Eire
}

\begin{abstract}
Aim: This study aimed to explore how the views of patients with coronary heart disease (CHD) could inform the design of an information booklet aimed at providing patients and practitioners with a resource to help influence positive health behavioural outcomes. Background: Coronary heart disease has major consequences in terms of patient suffering and economic costs, with morbidity and mortality figures in the United Kingdom and the Republic of Ireland among the highest in Europe. Lifestyle behaviours such as smoking, eating an unhealthy diet and a lack of exercise are strongly associated with an increased CHD risk, and practitioners report that health education materials are used in practice to help advise and educate patients about the consequences of their lifestyle. Methods: Opinions of patients with CHD were explored concerning their information needs, particularly lifestyle advice, using a qualitative approach in four general practices. This information was used to design a booklet for a pilot study aimed at promoting healthy lifestyle behaviours and medication adherence among people with CHD. Focus group discussions explored patients' opinions about the booklet's 'fitness for purpose'; semi-structured interviews with practitioners examined their views on the booklet's usefulness. Findings: In initial focus groups, patients identified gaps in their information provision regarding coping with stress, available local community support and medication purpose. Previously published literature was modified to address these gaps. Patients in the pilot study were satisfied with the re-designed booklet. Practitioners reported that its use in consultations enabled change implementation and facilitated patients' understanding of connections between lifestyle and health outcomes.

Acknowledging the opinions of CHD patients in producing health information booklets emphasized a patient-centred approach and therefore supported practitionerpatient partnerships for choosing healthy lifestyle choices.
\end{abstract}

Correspondence to: Claire S. Leathem, Northern Ireland Clinical Research Network (Primary Care), Level 4 Dunluce Health Centre, 1 Dunluce Avenue, Belfast BT9 7HR, UK. Email: c.leathem@qub.ac.uk

(C) 2009 Cambridge University Press 
Key words: coronary heart disease; health promotion communication; primary health care; qualitative research

Received 8 July 2008; accepted 12 April 2009

\section{Introduction}

Coronary heart disease (CHD) has major consequences, both in terms of human suffering and economic costs (BHF (British Heart Foundation), 2006), with CHD morbidity and mortality in the United Kingdom and the Republic of Ireland currently among the highest in Europe. A diagnosis of CHD necessitates patients attending frequent consultations in order to prevent further events and lessen potential complications. The general practitioner (GP), supported by the primary health care team, provides much of the care of these patients. Factors strongly associated with increased risk in CHD include lifestyle behaviours such as smoking, an unhealthy diet and lack of exercise. Practitioners have reported using written information contained in leaflets in order to instruct and educate patients about the health consequences of lifestyle (Beaver and Luker, 1997; Paul and Redman, 1997; Little et al., 2004).

Given that CHD patients have a wide choice of sources of information, including family members, the media, alternative practitioners and the Internet, health practitioners have a responsibility to ensure that they are provided with evidencebased, accurate, unbiased information which meets their individual need (Wilson, 2003). Earlier efforts to improve effectiveness of information leaflets have focused on enhancing their readability, using appropriate language and style and formulae which guide the construction of text in order to render health promotion messages simple enough to be understood by the largest audience (Beaver and Luker, 1997). However, readability formulae can overestimate the difficulty of medical terminology when patients are familiar with the language used to describe their illness (Smith et al., 1998; Rees et al., 2003): predictions of understanding and learning require reflection on the reader's prior knowledge (McNamara, 2001). Ability to utilize information, therefore, goes beyond factual content and design attributes to allow for factors that directly impact on the way patients cope with health information, including their current situation, past experiences and personality (Gal and Prigat, 2005).

Criticism has been levelled at the production of leaflets with an average reading age of nine, leading to a 'dumbing down' of information at the expense of content (Dixon-Woods, 2001). While adult literacy figures report almost $20 \%$ of the population in both UK and Ireland has some degree of problem with reading skills (IALS (International Adult Literacy Survey), 1998), there is concern that readability testing may contribute to the production of ineffective health-promotion literature by simplifying the information until it becomes meaningless to the people for whom it is intended. The ineffectiveness of literature is due to a failure to appreciate the value of patients' individual resources (Dixon-Woods, 2001) and the unique life experiences which they bring to the interpretation of information (Rogers, 2004).

Much patient information has been produced by health professionals and agencies ( Gal and Prigat, 2005), based on their collective knowledge of the topic, accepted guidelines for design and content and messages considered appropriate for their target patient audience (Kreuter et al., 2000). Modest benefits have been reported in the use of these materials, some of which utilize established theories of health behaviour change (Beaver and Luker, 1997; Little et al., 2004). However, few interventions outside the area of increasing medication concordance utilize models of testing and improvement to obtain the patients' perspective on information provision (Harris et al., 2005) and develop effective educational strategies.

Successful behaviour change materials are those which patients feel are relevant to them and contain practical encouraging information which is up to date or 'new' (Bull et al., 2001). Information that is tailored to patient need provokes positive responses and is more effective when the patient is motivated to implement a behavioural change. Practitioners can use their skills in motivational interviewing to help increase patients' receptivity to adopting healthier lifestyle choices. Patients who do not perceive a need for change, 
require information that raises consciousness and helps them to explore their ambivalence (Rollnick et al., 1999).

Earlier research has suggested that there is a need for those producing patient information, to challenge current thinking on recommended informational characteristics in order to increase the effectiveness of their printed materials (Paul et al., 2003). Few studies have attempted to elicit CHD patients' views regarding their perceived needs for lifestyle information or regarding the appropriateness of written materials planned for use in a research trial seeking evidence of best practice in chronic disease management. In this paper, we describe the process of developing an information booklet aimed at addressing patient information needs and helping to promote a more active patient role in consultations with practitioners. The booklet was developed as a resource for a randomized controlled trial (RCT) of an intervention to improve secondary prevention of CHD in primary care for people who were known to have CHD (Murphy et al., 2005). We aimed to explore CHD patients' perceived needs for lifestyle information, their perceptions of information they had received previously, practitioners' perceptions of available information and, within a pilot study involving use of the booklet we developed, patients' and practitioners' views of its value.

\section{Methods}

Using qualitative methodology, we explored the perceived information needs of patients with established CHD, particularly in relation to lifestyle. This is an appropriate method of data collection when inquiries relate to personal experiences of disease and perceptions of health belief and values. It provided an understanding of the issues of importance to both practitioners and patients (Kitzinger, 1995). The findings were used to develop an information booklet for use in patient-practitioner consultations (see Figure 1), to facilitate discussion of behaviour modification in relation to health consequences (Stubblefield and Mutha, 2002). Subsequently, we used the booklet in a pilot study and then, amongst the pilot study participants, we explored patients' opinions about its 'fitness for purpose', and practitioners' views on its usefulness.

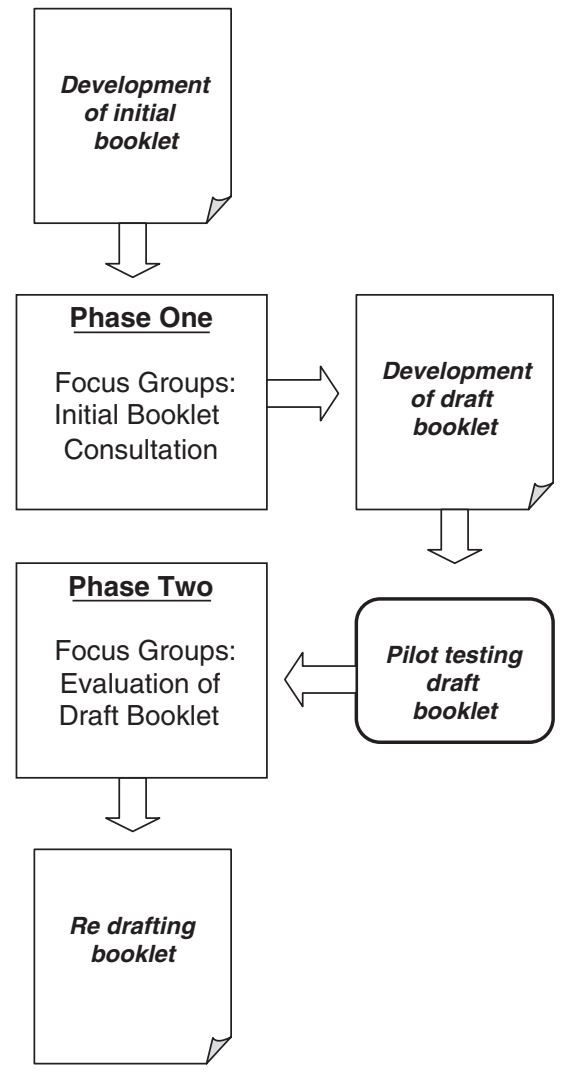

Figure 1 Flow chart of stages of the booklet development process

\section{Study setting}

This study was conducted in general practices in four sites, two located in Northern Ireland and two in the Republic of Ireland. This study had two phases; one prior to and another after a pilot of a RCT of an intervention which included the use of an information booklet, given to patients during consultations with practitioners whose role was to encourage and support healthy lifestyle behaviours and medication adherence.

\section{Sample}

\section{Phase 1}

In each practice, two focus groups, one involving staff and another involving patients, were held. Patients with diagnosed CHD, of no less 
than three months duration, were selected in each practice, using maximum variation sampling and including adults, both male and female, without age restriction. Patients who had a physical or mental illness preventing them from participating and engaging in self-selected lifestyle change were excluded. The selected patients were contacted by mail, inviting them to participate in a group discussion at their practice. The mailing included a patient information sheet, a consent form and a reply slip to record their decision with a stamped addressed envelope to post back to the practice. Non-responders were followed-up with a telephone call by the practice nurse two weeks later. All practice staff in each practice were invited to attend by liaison with the practice managers and GPs with the aim of holding discussions when the maximum number of staff members were on site. To facilitate attendance, staff focus groups were held during extended lunch times.

\section{Phase 2}

Following participation in the pilot study, 27 patients (who had not taken part in the initial focus groups) were invited to a focus group for feedback on the perceived usefulness of the booklet; 17 attended. Different patients were selected for the two sets of data collection in order to minimize the potential for bias and to facilitate access to a greater breadth of opinions regarding the booklet.

Practitioners, both GPs and practice nurses $(n=12)$, who had been involved in using the booklet in patient consultations, were asked to participate in one to one interviews in order to evaluate their experiences of using the booklet. Interviews facilitated detailed descriptions of their consultations. In total, 10 practitioners participated.

\section{Data collection}

Exploratory questions were designed to provide detail-rich information from patients, using their own language and terminology. They were asked open questions about their previous experiences of receiving lifestyle information related to $\mathrm{CHD}$ and any barriers they had encountered in receiving and using the information.
In Phase 1, practitioners' opinions were explored regarding their perceived barriers and facilitators to providing patients with secondary prevention information. In Phase 2, the views of both patients and practitioners on consultations involving the use of the booklet were sought, using questions focused on its usefulness in helping patients to plan and implement lifestyle changes. Possibilities for innovations in providing information relevant to CHD patients were explored.

The initial data collection with invited focus groups took place between March and June 2003. Following the pilot study, which involved at least two consultations for each participant, focus groups were held in March 2004. Two members of the research team were present at every focus group and the dialogue was audio taped before being transcribed verbatim. All focus groups lasted approximately $90 \mathrm{~min}$ and written notes were taken of observations of the focus group interactions (Corrigan et al., 2006).

\section{Ethical considerations}

Patients received a written explanation of the study and contact telephone numbers for the research nurse and project manager. The patient information sheets stated that they were free to refuse participation in both the focus groups and the pilot study, and that their decision not to participate would in no way affect the care they received at the practice. The focus groups and interviews were tape recorded with the participants' consent and assurances given that all information would remain confidential and anonymous. The research ethics committees of the Irish College of General Practitioners and the Queen's University Belfast approved the study.

\section{Data analysis}

Data analysis was conducted using the qualitative computer software programme NUDIST (N6) (Non-numerical Unstructured Data Indexing Searching and Theorizing). Transcripts were initially analysed separately by two different researchers to identify the main issues. Following discussions between the researchers, these were indexed and categorized using a thematic analysis framework. 


\section{Results}

\section{Phase one: initial focus groups}

Of the 60 patients invited to attend the initial focus groups, 23 attended. A total of 29 out of the 46 practice staff members invited took part (see Table 1). The findings directly relate to the information needs of patients; excerpts of candid dialogue demonstrate and reveal patient insights and experiences and practitioner perceptions of written information provision. We identified four themes relating to patients' and practitioners' perceptions of information provision relating to CHD: experience of information provision, impact of lifestyle information, need for information and support, and structured personalized information.

\section{Experience of information provision}

At this initial focus group, some patients said they were happy with the amount and content of information they had received after their cardiac event, whilst others stated that the information lacked detail. Patients had received varying numbers of leaflets, relating to a variety of topics. Some reported that they had no written information at all and others only had information that they had accessed themselves from newspapers, magazines and the Internet.

\section{Impact of lifestyle information}

Many patients had made a number of positive lifestyle changes since their cardiac event. Several talked about having taken up exercise such as walking, while others had made dietary modifications and cut down on smoking or drinking. Some reported that the information they had been given had made them more aware of the effect of lifestyle on their health. Others mentioned that the information had encouraged them to consider weight-loss and exercise as key behaviours to address:

It's (the information) made me slightly more health conscious.

\section{(Practice 4, male patient)}

Stress was perceived, both as a contributor to participants' cardiac problems and one of the most difficult lifestyle areas to alter. Some reported their own ways of reducing and coping with stress, but these were not always healthy options and did not reflect any positive lifestyle impact of information received in relation to other risk factors:

If I don't have those two cigarettes a day, I suffer more from stress so the balance is - I have the two cigarettes.

(Practice 3, female patient)

\section{Need for information and support}

Many indicated a gap in information regarding stress management, with comments that they had not received any relevant or helpful information in this area and that there was a need for help in regard to this:

...more important than your body is your... state of mind...there's not enough dealing with what goes on in your head.

(Practice 2, female patient)

The majority of patients acknowledged that they found it very difficult to adjust their lifestyle and that maintaining change was problematic. They valued information being provided by their own GP/practice nurse. They expressed a need for information to be tailored to their personal situation as opposed to receiving generic advice.

Table 1 Overall patient distribution at focus groups

\begin{tabular}{lll}
\hline & $\begin{array}{l}\text { Phase 1 }(n=23) \\
\text { Pre-booklet consultation } \\
\text { March-June 2003, } 4 \text { groups }\end{array}$ & $\begin{array}{l}\text { Phase 2 }(n=17) \\
\text { Evaluation of draft booklet } \\
\text { March 2004, } 4 \text { groups }\end{array}$ \\
\hline Male & $n=17(74 \%)$ & $n=11(65 \%)$ \\
Time since CHD diagnosis & 3 months-20 years & 4 months-23 years \\
Age & & $49-80$ years \\
Range & $48-74$ years & 64 \\
Mean & 61 & 49
\end{tabular}

$\mathrm{CHD}=$ coronary heart disease. 
This was emphasized particularly in relation to diet:

...someone said you better keep down the weight but nobody else tells me what I shouldn't do without or anything like that.

(Practice 3, male patient)

They also identified a failure of health service provision of information about local community resources for ongoing support in adopting or maintaining healthy lifestyle behaviours, and perceived value in having peer support:

There's no follow on from the (cardiac) rehabilitation...I mean exercise and the rehabilitation is great.... you're meeting people and you felt good after it ... but there was no follow up from that....

(Practice 3, female patient)

I think it'd be nice to meet up with other people who are similarly afflicted to see how they achieve their targets.

(Practice 2, male patient)

there's people round here would love to go for a walk at night if they'd someone to go with ... and if you knew who they were you could go out together ........

(Practice 3, female patient)

Participants also reported feeling that they needed more information about their medication; several were unclear about the purpose and effects of their prescribed medications:

I'm on 5 tablets a day now... I haven't a clue what they're for.

(Practice 4, male patient)

\section{Structured personalized information}

Practitioners talked about the significance of having a structure for giving patients consistent lifestyle information:

...that we are giving the same messages as regards lifestyle.... I think that's very important.

(Practice 2, GP)

While some health Internet sites were cited as sources of up to date information, insufficient practice resources sometimes played a part in limiting the provision of this information to patients:

you can print off leaflets for the individual
patient....... the problem is we have no
printer.

(Practice 2, GP)

Practitioners stated that written information was an important part of helping patients' self management:

Give them [patients] the information and show them how to manage things themselves....

(Practice 1, GP)

and also suggested how the format could contribute to helping behaviour change by:

...really personalising it and looking at where they [the patients] are and where they're coming from.

(Practice 4, practice nurse)

However, some practitioners doubted that patients would react positively to being asked to self-monitor between consultations, using a written structured format:

I just don't know how practical it is, I mean a stress self-monitoring form ..., I'd be interested to see how many people actually do fill that in.

(Practice 1, GP)

\section{Booklet design and pilot study}

Prior to the Phase 1 focus groups, an initial booklet had been drafted, following a review of health education leaflets produced by Health Promotion Departments and the British and Irish Heart Foundations. Theoretical approaches relating to language, graphics, colour, layout and local context were applied to its format and content. The initial booklet had not included information relating to stress management, medication or local community support information: it was revised, based on the themes identified from the focus groups. The booklet used in the pilot study was designed in A5 size format and divided into the following sections:

1. CHD medications

2. Smoking

3. Exercise

4. Healthy eating

5. Stress 
6. Local community support (for facilitating healthy lifestyles, eg, exercise or weight control community groups for mothers and pensioners).

Pages for recording medications, goal setting, action plans and 'diary' type self-monitoring were included, allowing patients to record personal targets and plans and to actively assess their progress. Within a pilot study (Murphy et al., 2005) patients were invited by practitioners during consultations to identify, from the revised booklet, a lifestyle behaviour for change and, in collaboration with the practitioner, to set a goal and plan of action. This plan was recorded in the booklet which they were asked to bring to further consultations to review progress.

\section{Phase two: evaluation of the pilot booklet}

Following the pilot study, 17 of the 27 patients invited attended focus groups (see Table 1). Of the 12 practitioners invited to the one-to-one interviews, 10 attended (one nurse had left the practice and one practice manager was on leave). Participants' views regarding the booklet and its use related to three themes - the impact of lifestyle information, goal setting and recording, and the booklet format.

\section{Impact of lifestyle information}

Participants reported having found the booklet useful, particularly in explanations of links between lifestyle and heart disease, and the pictorial guide to healthy eating and information on coping with stress. Some patients stated they had received similar information previously but felt the booklet had additional value:

this is reinforcing the information that I had ....

(Practice 2, male patient)

I found it very informative ... very good ... ... .

(Practice 3, male patient)

Conversely, others denied receiving similar information previously.

Comments made regarding information about stress highlighted the importance of having listened to patients' perspectives in the initial focus groups:

The part about the stress I found very helpful... - ways of steering clear of stress and how to cope with it, because I found it very very hard to accept this whole change in my lifestyle... from being out working fulltime ... do you know what I mean?

(Practice 1, female patient)

The usefulness of the section on the nature and purpose of CHD medications was mentioned, particularly among patients who took a large number of medications:

...because the house is like a chemist's shop .......

(Practice 1, male patient)

This page for patients' medications to be recorded provided practitioners with an opportunity to explain the purpose of the various drugs and was appreciated by patients:

she [the practitioner] explained everything ... what they were all for ... and what your aspirin was for .... and about the ones to lower your cholesterol ....

(Practice 1, female patient)

It was nice getting a refresher ... getting it all down on paper sometimes helps ....

(Practice 2, male patient)

Patients valued information provision relating to local community support:

...its good back up [having information on local amenities]....

(Practice 1, female patient)

\section{Goal setting and recording}

Participants were reluctant to complete food diaries or the self-monitoring pages and reported that they felt they were time-consuming and unhelpful:

...trying to fill that smoking bit in everyday would put the cigarettes in my mind.

(Practice 3, female patient)

However, they valued the booklet structure for working with the practitioner to identify small achievable changes:

I don't normally take a lot of fruit but I've been taking a bit more lately.

(Practice 2, male patient) 
...now walking more instead of watching TV [television] that sort of thing.

(Practice 2, male patient)

I take more vegetables for my dinner.

(Practice 2, female patient)

Practitioners appreciated having a structure within the booklet which allowed them to facilitate patients to implement their chosen lifestyle changes. Several reported having incorporated it into their practice:

... so I think the book as a template to go through things for myself ... to remind me of things would be excellent....

(Practice 1, Nurse)

... I think its good to help me focus as a nurse...... you know the way sometimes if you're sitting here doing a healthy heart clinic in the morning I think we tend to focus too much on bloods... what your blood is ...... and what your blood pressure is ....... made patients think you know about what they were doing in their diet and stuff like that.

(Practice 2, Nurse)

I've used it just as a guidance for some of the patients... and just for me ...for my benefit.

(Practice 1, Nurse)

I went through the booklet with each of them......filled in bits and pieces then homed in on one chapter with them that they have identified that they wanted to work on.

(Practice 4, GP)

\section{Booklet format}

Both patients' and practitioners' comments in response to questions regarding possible improvements to the format of the booklet indicated its acceptability. The only adverse comment identified was that the font was too small:

Its format is ...very clear .......

(Practice 1, male patient)

Its format is handy ...you can go back and look at it.

(Practice 3, male patient)

I didn't find anything actually wrong with it ....

(Practice 3, male patient)
Participants suggested that information should be made as personal as possible to individuals and could contain a list of common heart disease terms and diagrams that would be useful. Some patients would have been happy to have received additional detail within the information provided:

... maybe a diagram of the heart and valves ...explain what a stent is .... things like that that people don't know.

(Practice 2, male patient)

Some patients mentioned more pictures could be included and a 'frequently asked questions and answers' section:

I know the Doctors probably do this anyway but it would help us if it [personal cholesterol and blood pressure measurements] was recorded so that we could read it to see how we are making progress.

(Practice 2, male patient)

You could put a lot more in that book so you could.

(Practice 2, male patient)

\section{Discussion}

This study used qualitative methodology to explore and afford insights into patients' beliefs, attitudes and understanding of lifestyle information. The findings affirmed the value of using patients' opinions in informing the design of information used in consultations for secondary prevention for patients with CHD. Participants' previous experience of information provision varied, but the study showed that patients valued information given by their own practitioners and perceived a need for information which was personalized to their own circumstances, particularly in relation to stress, medication and other sources of support. Those who used the booklet - which was revised to include this information - confirmed appreciation of its provision. However, they varied in the extent to which they wished to be involved in recording information about their progress towards achieving lifestyle change and in the detail of information desired.

\section{Comparison with previous work}

The current findings provide evidence of the range of individual perceptions and circumstances 
which may influence patients' needs for information and support relating to lifestyle change. Earlier work has shown that in order to make the link between lifestyle information and the instigation of a behaviour change, patients need to believe they have a measure of control over their lives (Morrall, 1996; Feldman-Stewart et al., 2007). Motivation to change lifestyle or take prescribed medication appears to be prompted more easily in those patients who are experiencing cardiac symptoms such as chest pain (Little et al., 2004). Understanding individual attitudes to changing behaviour must be part of the support strategy used by practitioners in patient consultations, leading to emphasis on information of particular relevance for that person. Facilitating patients to become actively involved in their care has been closely linked to the holistic approach to patient care (Rodgers, 1989), and studies have shown that patients who are involved in their treatment decisions have higher patient satisfaction, better outcomes and emphasize the benefits of collaborative relationships in empowering patients to achieve mutually agreed goals (Friere, 1990; Little et al., 2001; Haidet et al., 2006). The challenge of providing effective patient information involves both practitioners and patients. The practitioner provides medical expertise including the ability to correct patients' misinterpretation of information and the patient contributes their unique experiences of living with a disease and taking medications (Frank, 1995). Barriers to effective patient information provision, for secondary prevention for CHD, need to be addressed in order to close the gaps between planning and implementing changes for modification of risk factors, and allow patients to gain years of health that would otherwise be denied them.

\section{Study strengths and limitations}

A strength of obtaining information through focus groups is that it may allow patients to broaden the focus on the topic planned for discussion as they talk to each other, ask questions and express their opinions (Morgan, 1997). The focus groups proved difficult to assemble, many invited patients cancelled or did not attend, perhaps because they did not wish to express personal information in a group or because, as meetings were only arranged during the day they may have been unable to get time away from their work. However, maximum variation sampling was used to include a wide range of patient views. Initial focus groups included a high proportion of men $(74 \%)$, but this tended to reflect the higher prevalence of CHD amongst men and whilst there may be differences in the perceived information needs of men and women with CHD, both were included in the discussions. We recognize that the findings reported relate to qualitative information only and that we have not attempted to use a quantitative measure of patient satisfaction to evaluate the booklet we designed. This however, will be assessed at a later date within the definitive Secondary Prevention of Heart Disease in General Practice (SPHERE) trial.

\section{Conclusion}

Our study showed that utilizing the opinions of CHD patients is of value in developing appropriate materials of relevance to the process of lifestyle modification. Without patients' input, the information materials used in the RCT would not have afforded maximal potential for effecting optimal implementation of secondary prevention. No-one has yet identified the design factors or semantic attributes which could render information both compelling and comprehensible to every reader (Friedman and Hoffman-Goetz, 2006). While it may not be possible for practitioners to develop a booklet, specifically adapted for use with their own patients, they can seek patients' views regarding their needs for information and respond by attempting to provide appropriate 'fit for purpose' information.

\section{Practice implications}

Providing information with accurate messages reflecting patients' perceived information needs will assist patients to put their change decisions into action. Support to effect and maintain change can be provided by practitioners in patient-centred consultations and supplemented by information about the support structures and facilities available within their locality. This recognizes that patients have a stake in the information created for them and shows consideration of their individual agenda. Using a 
structured approach to providing information which is personalized to individuals' circumstances may help practitioners to facilitate patients with CHD to achieve better health outcomes.

\section{Acknowledgements}

We would like to acknowledge the Health Research Board (Ireland) and the Irish Heart Foundation as the funding sources which supported the research.

MEC is co-funded by the Centre of Excellence for Public Health (Northern Ireland), a UKCRC Public Health Research Centre of Excellence. Funding from the British Heart Foundation, Cancer Research UK, Economic and Social Research Council, Medical Research Council, Research and Development Office for the Northern Ireland Health and Social Services and the Wellcome Trust, under the auspices of the UK Clinical Research Collaboration, is gratefully acknowledged.

We would like to sincerely thank Ms Pauline Clerkin for her valuable contribution to the focus group interviews and the patients and practice staff in each of the participating practices.

\section{References}

Beaver, K. and Luker, K. 1997: Readability of patient information booklets for women with breast cancer. Patient Education and Counseling 31, 95-102.

BHF (British Heart Foundation). 2006: Coronary heart disease statistics. Retrieved from http://www.heartstats.org/datapage.asp?id=5712

Bull, F.C., Holt, C.L., Kreuter, M.W., Clark, E.M. and Scharff, D.P. 2001: Understanding the effects of printed health education materials: which features lead to which outcomes? Journal of Health Communication 6, 265-79.

Corrigan, M., Cupples, M.E., Smith, S.M., Byrne, M., Leathem, C.S., Clerkin, P. and Murphy, A.W. 2006: Developing a complex intervention for secondary prevention of coronary heart disease in two different healthcare systems: the contribution of qualitative methods. BMC Health Services Research 6, 90. Available at http://www.biomedcentral.com/1472-6963/6/90

Dixon-Woods, M. 2001: Writing wrongs? An analysis of published discourses about the use of patient information leaflets. Social Science \& Medicine 52, 1417-432.

Feldman-Stewart, D., Brennenstuhl, S. and Brundage, M.D. 2007: A purpose-based evaluation of information for

Primary Health Care Research \& Development 2009; 10: 189-199 patients: an approach to measuring effectiveness. Patient Education and Counseling 65, 311-19.

Frank, E. 1995: Discussion alliance not compliance: a philosophy of outpatient care. Journal of Clinical Psychiatry 56, 16-17.

Friedman, D.B. and Hoffman-Goetz, L. 2006: A systematic review of readability and comprehension instruments used for print and web-based cancer information. Health Education \& Behavior 33, 352-73.

Friere, P. 1990: Pedagogy of the oppressed. New York: Continuum Press.

Gal, I. and Prigat, A. 2005: Why organizations continue to create patient information leaflets with readability and usability problems: an exploratory study. Health Education Research 4, 485-93.

Haidet, P., Kroll, T.L. and Sharf, B.F. 2006: The complexity of patients participation: lessons learned from patients' illness narratives. Patient Education and Counseling 62, 323-29.

Harris, M., Smith, B. and Veale, A. 2005: Printed patient education interventions to facilitate shared management of chronic disease: a literature review. Internal Medicine Journal 12, 711-16.

IALS (International Adult Literacy Survey). 1998: Retrieved 2 October 2006 from http://www.statcan.ca/english/Dli/ Data/Ftp/ials/ials1994-96-98.htm

Kitzinger, J. 1995: Qualitative research: introducing focus groups. British Medical Journal 311, 299-302.

Kreuter, M.W., Oswald, D.L., Bull, F.C. and Clark, E.M. 2000: Are tailored health education materials always more effective than non-tailored materials? Health Education Research 15, 305-15.

Little, P., Everitt, H., Williamson, I., Warner, G., Moore, M., Gould, C., Ferrier, K. and Payne, S. 2001: Observational study of effect of patient centredness and positive approach on outcomes of general practice consultations. British Medical Journal 323, 908-11.

Little, P., Dorward, M., Warner, G., Moore, M., Stephens, K., Senior, J. and Kendrick, T. 2004: Randomised controlled trial of effect of leaflets to empower patients in consultations in primary care. British Medical Journal $328,441$.

McNamara, D.S. 2001: Reading both high and low coherence texts: effects of text sequence and prior knowledge. Canadian Journal of Experimental Psychology 55, 51-62.

Morgan, D.L. 1997: Focus groups as qualitative research, second edition. London: Sage.

Morrall, P. 1996: Clinical sociology and the empowerment of clients. Mental Health Nursing 16, 24-27.

Murphy, A.W., Cupples, M.E., Smith, S.M., Byrne, M., Leathem, C.S. and Byrne, M.C. 2005: The SPHERE study. Secondary prevention of heart disease in general practice: protocol of a randomised controlled trial of tailored practice and patient care plans with parallel qualitative, economic and policy analyses. Current 
Controlled Trials in Cardiovascular Medicine 6, 11. Available at http://cvm.controlled-trials.com/content/6/1/11

Paul, C.L., Redman, S. and Sanson-Fisher, R.W. 2003: Print material content and design: is it relevant to effectiveness? Health Education Research 18, 181-90.

Paul, C.R. and Redman, S. 1997: A review of the effectiveness of print material in changing health related knowledge, attitudes and behaviour. Health Promotion Journal of Australia 7, 91-99.

Rees, C.E., Ford, J.E. and Sheard, C.E. 2003: Patient information leaflets for prostate cancer: which leaflets should healthcare professionals recommend? Patient Education and Counseling 49, 263-72.

Rodgers, B.L. 1989: Concepts, analysis and the development of nursing knowledge: the evolutionary cycle. Journal of Advanced Nursing 14, 330-35.
Rogers, R. 2004: Storied selves: a critical discourse analysis of adult learners' literate lives. Reading Research Quarterly 39, 272-305.

Rollnick, S., Mason, P. and Butler, C. 1999: Health behaviour change: a guide for practitioners. Edinburgh: Churchill Livingstone.

Smith, H., Gooding, S., Brown, R. and Frew, A. 1998: Evaluation of readability and accuracy of information leaflets in general practice for patients with asthma. British Medical Journal 317, 264-65.

Stubblefield, C. and Mutha, S. 2002: Provider-patient roles in chronic disease management. Journal of Allied Health 31, 87-92.

Wilson, V.L. 2003: Survey of information and support needs of people with type I diabetes. Journal of Diabetes Nursing. Retrieved on 1 August 2006 from http://www.findarticles.com/ articles/mi_m0MDR/is_7_7/ai_n6100328 